\title{
Improving effectiveness of scoliotic posture prevention and correction in primary school children
}

\author{
Artem Moment ${ }^{1 *}$, Denis Semyonov ${ }^{2}$, Elena Karpenko ${ }^{1}$, Irina Kolbasova ${ }^{1}$, Lena \\ Rubenkova ${ }^{1}$, and Elena Tarasova \\ ${ }^{1}$ Health Protection and Physical Culture Chair, Institute of Education and Social Studies, Pskov State \\ University, Pskov, Russia \\ ${ }^{2}$ Theory and Methodology of Gymnastics Chair, Velikiye Luki State Academy of Physical Education \\ and Sports, Velikiye Luki, Russia
}

\begin{abstract}
For several decades the problem of prevention and correction of posture disorders in primary school children has not lost its relevance. Statistics show that $68-94 \%$ of primary school students have posture disorders. A special place in the classification of posture disorders is occupied by a scoliotic posture. Such a statement indicates that healthimproving physical education does not fully solve the tasks assigned to it. Analysis of literature sources allowed us to establish two main reasons for the low effectiveness of prevention and correction of scoliotic posture in primary school children. The first reason relates to the low effectiveness of early diagnosis scoliotic posture, and the second reason is low overage of the aspects of the problem, revealing the model of periodization of fitness training with the children in the theory and practice of physical culture. The results of previous studies have allowed us to suggest ways to improve the effectiveness of prevention and correction of scoliotic posture in primary school children, namely: to expand the range of traditional methods of early diagnosis of scoliotic posture through motor and postural diagnostics; to use a nonlinear model of periodization of physical activity to create optimal conditions for leveling muscle imbalance and the formation of rational motor automatisms and patterns in the context of health training.
\end{abstract}

\section{Introduction}

Improving the effectiveness of implementing the task of preserving and improving the health of students in educational institutions has been an important area of research for several decades. The fundamental role in solving this basic task is assigned to physical education, which is carried out through specially organized forms of physical exercise, integrated into the educational process and taken out of it. Researchers have shown that students in grades 2-4 have significantly lower postural muscle endurance indicators than first-graders [1], which is a predictor of muscle imbalance [2].

* Corresponding author: ar.moment $@$ yandex.ru 
The data from scientific research over the past 15 years indicate a low level of health in primary school children [3-6]. Close attention of a wide range of specialists is aimed at solving problems of prevention and correction of posture disorders in children of primary school age [7-11]. A special place is occupied by violations of posture in the frontal plane, also called a scoliotic posture, scoliotic posture anomaly and school scoliosis [12-15].

According to the latest data, the prevalence of posture disorders in primary school children is in the range of $68-94 \%[12,16-18]$. Such a statement indicates that healthimproving physical education does not fully solve the tasks assigned to it.

The purpose of the study is to supply existing ideas about the prevention and correction of scoliotic posture in primary school children.

The research objectives are:

1. To determine the main reasons for the low effectiveness of prevention and correction of scoliotic posture in primary school children.

2. To suggest the ways to improve the effectiveness of prevention and correction of scoliotic posture in primary school children.

\section{Materials and methods}

The search for the reasons for the low effectiveness of prevention and correction of scoliotic posture in primary school children involved the analysis and systematization of scientific research data over the past 15-20 years on the subject under consideration.

The proposed recommendations aimed at improving the effectiveness of prevention and correction of scoliotic posture in primary school children are based on previously conducted original research by the first author of this article.

\section{Results and discussion}

Analysis of the current state of the problem of prevention and correction of scoliotic posture in primary school children allowed us to identify two objective reasons for the low effectiveness of these measures.

The first reason is the low effectiveness of early diagnosis of scoliotic posture in primary school children using traditional screening methods, which rely mainly on visual assessment of the symmetry of posture in a static body position [19-21].

A number of modern studies devoted to the problem of postural disorders in primary school students [22, 23] show that this group has difficulties in maintaining a vertical posture, as well as asymmetry in movements [24, 25]. In both cases, the objective factor causing these phenomena is considered to be an imbalance of postural muscles.

Therefore, the use of the methods for analyzing the parameters of postural function and evaluating the symmetry of movements can be an effective method for early diagnosis of scoliotic posture. However, at the moment, indicators of postural function and a set of diagnostic movements with high validity in the context of scoliotic posture diagnostics are not defined.

In previous studies [2, 26], attempts were made to identify the most valid parameters of postural function and the range of motor tasks in the context of early diagnosis of scoliotic posture in primary school children. As a result, it was found that not all parameters of the postural function are highly reliable. For example, the postural function parameter, which is often used by researchers and reflects the area of the statokinesiogram (S, mm2), showed low reliability in the 20 -second Romberg test. The rate of the speed of movement of the pressure center $(\mathrm{V}, \mathrm{mm} / \mathrm{s})$, coefficient of abrupt change in the direction of movement $(\%)$, deviation of the center of pressure in the frontal plane $(\mathrm{Q}(\mathrm{x}) \% \mathrm{~mm})$ and the integral 
indicator of the quality of the equilibrium function (\%) reflect negative changes in the postural system caused by muscle imbalances and can be used as informative criteria for evaluating the effectiveness of interventions for the prevention and correction of scoliotic posture.

A simpler, from the point of view of the instrumentation used, but no less promising method for early diagnosis of scoliotic posture in younger schoolchildren is a visual assessment of the symmetry of the fundamental (basic) movements. Such methods in health-improving physical education and fitness are gaining more and more popularity [25]. The general concept of motor diagnostics methods is to assess the effectiveness of fundamental movements fulfillment by means of a qualitative analysis of the technique of performing specially selected motor exercises. It has been experimentally proved that for children with scoliotic posture, when performing movements associated with flexion and extension of the trunk, hips and shoulders, asymmetries are manifested significantly more often than for children without such deviations [2, 12,24]. This fact allows us to consider these techniques as valid in relation to the early diagnosis of scoliotic posture.

In this regard, when organizing the process of physical education of primary schoolchildren and assessing its effectiveness, it is necessary to analyze not only the dynamics of quantitative values of indicators reflecting the level of development of the child's motor abilities, but also the qualitative forms of performing fundamental movements, and the nature of motor automatisms (posture, walking, running).

The second reason is insufficient presentation in the theory and practice of health related physical culture of the aspects of the issue, revealing the models of periodization of health related training with this category of children, in which the parameters of physical activity would be determined in the framework of the short and long term and the individual motor characteristics of children with scoliotic posture would be taken into account.

Muscular imbalance, which, in our opinion, causes such disorders, contributes to the destabilization of motor automatisms and patterns. The formed complex of non-optimal motor automatisms, against the background of developing muscular imbalance, does not allow to fully reveal the health-improving potential of physical exercises [24, 27]. While specialists in order to prevent and correct posture disorders mostly focus on the selection of corrective and preventive exercises [28-31], and to a lesser extent on planning and periodization of health related training, taking into account the influence of negative factors of muscle imbalance on the motor sphere of children.

In this regard, the need for periodization of health related training for younger schoolchildren with scoliotic posture is obvious. Taking into account the above, a model of periodization of health-improving training has been developed for this contingent.

The basis for the organization of health-improving and correctional classes with younger schoolchildren with postural disorders was health-improving gymnastics, consisting of consecutive 4-week blocks: the first week is an introductory block, the second and third week is a developmental / stimulating block, and the fourth week is a stabilizing / transitional block. It is possible both to use a set of successively replaced blocks, in which the complexity of exercises increases from one to another, and one block within the framework of other types of health-improving and sports training.

The features of the proposed method of prevention and correction of scoliotic posture in younger schoolchildren are:

- use of motor diagnostics methods within the framework of operational, current and staged control;

- step-by-step mastering and application of symmetrical dynamic, static-dynamic and static strengthening exercises of increasing complexity for the main muscle groups; 
- an individual approach to dosing physical activity when performing strengthening exercises using the method of standard-repetitive, interval exercises and adaptive learning techniques;

- non-linear model of periodization of physical activity.

Such a model takes into account the main features of the influence of muscle imbalance on the motor sphere of children, and also programs optimal conditions for leveling muscle imbalance and the formation of rational motor automatisms and patterns. Therefore, this model can be used in the context of organizing measures for the prevention and correction of scoliotic posture.

\section{Conclusion}

As a result of the study, it was revealed that at the moment there are two main reasons for the low effectiveness of measures for the prevention and correction of scoliotic posture in younger students.

The first reason is the imperfection of traditional methods of early diagnosis of scoliotic posture. The second reason is insufficient presentation in the theory and practice of health related physical education of models of periodization of health related training with younger schoolchildren with scoliotic posture.

It is worth noting that there are many more reasons why the statistics of posture disorders in younger schoolchildren have been deteriorating over several decades. However, many reasons associated with social and other factors are not within the competence of physical education specialists and therefore are not considered within the framework of this article.

Thus, in order to increase the effectiveness of the prevention and correction of scoliotic posture in children of primary school age, it is advisable:

- to supplement the range of traditional methods of early diagnostics of scoliotic posture with motor and postural (if possible) diagnostics within the framework of operational, current and staged monitoring;

- to use a non-linear model of periodization of physical loads to create optimal conditions for leveling muscle imbalance and the formation of rational motor automatisms and patterns.

\section{References}

1. I.V. Ryabova, Scientific notes of the P.F. Lesgaft University: scientific and theoretical journal, 2 (168), 300-306 (SPb., P.F. Lesgaft SSU, 2019) (In Russ.)

2. A.V. Moment, Scientific notes of the P.F. Lesgaft University: scientific and theoretical journal, 3 (157), 225-229 (SPb., P.F. Lesgaft SSU, 2018) (In Russ.)

3. L.I. Gabdrahmanova, Health prophylactics and physical preparation correction of primary school children by methods of Profilaktika zabolevaemosti i korrekciya fizicheskoj physical therapy Diss....Cand.Ped. (2009) (In Russ.)

4. N.N. Zinyakov, Kuban scientific medical bulletin №8. 88 - 90 (2009) (In Russ.)

5. V.N. Kochomanova, Problems and prospects of the formation of a healthy lifestyle in the information society: proceedings of the international scientific and practical conference, 100-103 (Irkutsk, 2016) (In Russ.)

6. O.A. Polikarpova, V.A. Kashuba, Y.V. Kozlov, T.A. Shitikov Physical culture, education, health: international conference paper VLGIFK December 12-13 2001, 164-168 (2002) (In Russ.) 
7. D.N. Artemov, Diagnostics and correction of schoolchildren posture diss.abstract...Cand.Med.Sc. (2005) (In Russ.)

8. A.M. Volkov, V.V. Popov, S.M. Chechel'nickaya, S.A. Orlenko, Bulletin of restorative medicine №4 (26). 60 - 64 (2008) (In Russ.)

9. M.S. Gorbachev // Physical education in school № 8. 25-28 (2005) (In Russ.)

10. T.G. Kovalenko, A.P. SHklyarenko, D.A. Ul'yanov, YU.G. Degtyarenko, Theory and practice of physical education № 10. 101 - 106 (2015) (In Russ.)

11. V.N. Kochomanova, Problems and prospects of the formation of a healthy lifestyle in the information society: proceedings of the international scientific and practical conference, 100-103 (Irkutsk, 2016) (In Russ.)

12. E.I. Aukhadeyev, Physical education in prevention, treatment, rehabilitation: a scientific and practical journal, 3-4(34-35), 92-95 (2010) (In Russ.)

13. A.D. Kashin, Skoliosis and posture disorders (2000) (In Russ.)

14. O.A. Polikarpova, Prevention of postural disorders by means of gymnastics among junior pupils based on individual approach: Diss....Cand.Ped. (2007) (In Russ.)

15. A. Moment, D. Semenov, V. Golubkov, O. Mushtukova, M. Semyonova, First International Scientific-Practical Conference "Actual Issues of Physical Education and Innovation in Sports" (PES 2020): BIO Web Conf Volume 26. (2020) URL: https://doi.org/10.1051/bioconf/20202600034 (accessed 02.11.2020).

16. N.N. Zinyakov, Kuban scientific medical bulletin №8. 88 - 90 (2009) (In Russ.)

17. M.O. Kuzmina, Healthcare, education and safety, 3 (11), 68-75 (2017) (In Russ.)

18. L. Yang, X. Lu, B. Yan, Y. Huang, iScience, 23(5), 101043 (2020)

19. F. Altaf, J. Drinkwater, K. Phan, A.K. Cree, Spine Deform, 5(5), 303-309 (2017)

20. T. Karachalios, J. Sofianos, N. Roidis, G. Sapkas, D. Korres, K. Nikolopoulos, Spine (Phila Pa 1976), 24(22), 2318-2324 (1999).

21. R. Ningthoujam, Int J Phys Educ Health Sports Sci, (3), 2-8 (2014)

22. V.V. Koss, Early diagnostics and prevention of progression of postural disorders and scoliosis of I-II degree among children in educational institutions: diss.abstract...Cand.Med.Sc. (2009) (In Russ.)

23. A.V. Moment, D.V. Semenov, Scientific notes of the University named after P.F. Lesgaft, 9 (139) 106 - 112 (2016) (In Russ.)

24. A.P. Shklyarenko, Physiological substantiation of Physical Education for scoliotic disease among children and teenagers: Diss....PhD in Biol. (2002) (In Russ.)

25. G Cook, L Burton, BJ Hoogenboom, M Voight, Int J Sports Phys Ther. 9(3) 396-409 (2014)

26. A.V. Moment, D.V. Semenov, Scientific notes of the University named after P.F. Lesgaft, 9 (139) 106 - 112 (2016) (In Russ.)

27. Ju.I. Dan'ko Essays on the physiology of exercise (1974) (In Russ.)

28. T.I. Zubkova, Theory and practice of physical culture. 67 (2008) (In Russ.)

29. A.N. Kudyasheva Physical rehabilitation of postural disorders in children of primary school age: dis. ... cand. ped. sciences (2012) (In Russ.)

30. M.A. Stepkina, V.K. Fedotov, A.P. Shklyarenko, System of diagnostic and conservative treatment measures for posture disorders and spinal deformities in children and adolescents (2009) (In Russ.) 
31. D.V. Erdenko, S.N. Popov, O.V. Kozyreva, XII International Scientific Congress "Modern Olympic and Paralympic Sports and Sports for All" (2008) (In Russ.). 